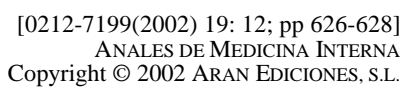

Copyright $($ ) 2002 ARAN EDICIONES, S.L.

An. Med InTERna (Madrid) Vol. 19, N. ${ }^{\circ} 12$, pp. 626-628, 2002

\title{
Eje hipófiso-suprarrenal en el alcoholismo crónico
}

\author{
E. VALLINA ÁLVAREZ, P. VILLAVERDE AMIEVA, J. RODRÍGUEZ SÁNCHEZ, \\ M. GALLEGO VILLALOBOS, E. GONZÁLEZ FERNÁNDEZ, \\ J. M. ARRIBAS CASTRILLO
}

Cátedra de Patología Médica. Hospital Central. Universidad de Oviedo

\author{
HYPOPHYSO-ADRENAL AXIS IN PATIENTS WITH CHRONIC \\ ALCOHOLISM
}

\begin{abstract}
RESUMEN
Objetivos: Valorar la situación del eje hipófiso-suprarrenal en pacientes con alcoholismo crónico.

Método: Se estudiaron 24 pacientes alcohólicos crónicos ingresados en nuestro centro en situación de delirio agudo.

Se determinaron cortisolemia a.m. y p.m. y niveles de ACTH a.m. junto a serie roja con sus índices de Wintrobe, número de plaquetas, trigliceridemia, colesterolemia total y HDL y actividad de transaminasas, fosfatasa alcalina y gammaglutamiltranspeptidasa en el suero.

Resultados: Las cifras medias de ACTH a.m. fueron de $41.2 \mathrm{pcg} / \mathrm{ml}$ para valores normales de $10-80 \mathrm{pcg} / \mathrm{ml}$, las de cortisolemia a.m. de 22 $\mathrm{mcg} / \mathrm{ml}$ (rango normal $5-25 \mathrm{mcg} / \mathrm{ml}$ ) y las de cortisolemia p.m. de 12.3 $\mathrm{mcg} / \mathrm{ml}$ (tango normal 5-15 $\mathrm{mcg} / \mathrm{ml}$ ).

Conclusiones: Las cantidades de alcohol ingeridas por los pacientes alcohólicos no parecen equiparables a las utilizadas y a las que condicionan una hiperfunción suprarrenal en la experimentación animal.

Los niveles de ACTH en sangre y de cortisolemia no son útiles como parámetros indirectos de alcoholismo. Al respecto, destacan como más efectivos los índices de Wintrobe y la actividad de gammaglutamiltranspeptidasa en el suero.
\end{abstract}

PALABRAS CLAVE: Alcohol. Marcadores. Eje hipófiso-suprarrenal.

\begin{abstract}
Objetives: To study the situation of the hypophyso-adrenal axis in patients with chronic alcoholism.

Method: 24 patients with chronic alcoholism admitted in our Institu tion with acute delirium were studied.

Cortisol am and pm und (ACTH levels, along with hemogram with Wintrobe indexes, patelets number, triglicerides, total and HDL choles terol, transaminases, alkaline phosphatase and gammaglutamyltrans peptidase were measured in serum.

Results: The mean values of ACTH am were $41.2 \mathrm{pcg} / \mathrm{ml}$ (normal values $10-80 \mathrm{pcg} / \mathrm{ml}$ ), of plasma cortisol am $22 \mathrm{mcg} / \mathrm{ml}$ (normal values $10-24 \mathrm{mcg} / \mathrm{ml}$ ) and plasmo cortisol pm $12.3 \mathrm{mcg} / \mathrm{ml}$ (normal values 5-12 $\mathrm{mcg} / \mathrm{ml})$.

Conclusions: The amount of alcohol consumed by alcoholic patients ist not comparable to that used in animal models, which induced adrenal hyperfunction.

Blood ACTH and cortisol levels are not useful as indirect markers of alcoholism. The Wintrobe indexes and serum gammaglutamyltranspepti dase levels are more useful for that purpose.
\end{abstract}

KEY WORDS: Alcohol. Markers. Hypophyso-adrenal axis.

Vallina Alvarez E, Villaverde Amieva P, Rodríguez Sánchez J, Gallego Villalobos M, González Fernández E, Arribas Castrillo JM. Eje hipófiso-suprarrenal en el alcoholismo crónico. An Med Interna (Madrid) 2002; 19: 626-628.

\section{INTRODUCCIÓN}

En 1960 Kissin y cols.(1) comunicaron que en los alcohólicos crónicos se incrementaban los valores de los 17-hidroxicorticoides en el plasma. Este mismo efecto fue observado también en las ratas tras la intoxicación aguda alcohólica por Kalant y cols., en 1963 (2).

En 1976 Smals y cols. (3) describieron el síndrome pseudocushingoide de los alcohólicos con aspecto externo indistinguible del síndrome de Cushing y con elevaciones de las corti- solemias que volvían a la normalidad a las 3 semanas de la interrupción de la administración de alcohol. Otros autores, como Rees (4) y Frajria y cols. (5), confirmaron posteriormente la existencia del síndrome pseudocushingoide alconólico.

En 1994 Cubelos (6) demostró en ratas sometidas a administración alcohólica crónica una elevación de los parámetros histio-fisiológicos de la actividad celular en las diferentes capas de la corteza suprarrenal.

Los mecanismos, por los que se podría explicar el síndrome Pseudo-Cushing de los alcohólicos sería:

Trabajo aceptado: 18 de septiembre de 2002

Correspondencia: Emilio Vallina Álvarez. Sección de Medicina Interna. Universidad de Oviedo. C/ Celestino Villamil, s/n. 33006 Oviedo. 
1. La alteración del funcionalismo hepático, lo que daría lugar a una deficiente inactivación de los glucocorticoides por estar disminuidas las actividades del citocromo P-450 y la reductasa del anillo A corticoideo.

2. La acción directa del alcohol sobre las glándulas suprarrenales. Coob y cols. (7) en 1981 perfundieron con alcohol etílico un cultivo de células suprarrenales, demostrando luego un aumento de esteroides en el medio en el que fueron cultivadas.

3. Un teórico incremento en la producción de GIP (péptido inihibidor gástrico, que estimularía unos receptores que se mostrarían anormalmente en la células suprarrenales). La razón de designar como "teórico" este incremento está en que tal mecanismo, demostrado en otros casos de síndrome de Cushing, no está probado en el Pseudo-Cushing alcohólico (8).

4. Un estímulo del eje hipotálamo-hipofisario $(8,9)$.

Tras la consideración de estos mecanismos patogénicos posibles de la hiperfunción suprarrenal se comprende que en las exploraciones analíticas de los pacientes alcohólicos puedan ser encontrados incrementos en las determinaciones de las cortisolemias a.m. y p.m. y variaciones en los niveles de ACTH que estarían disminuidos cuando prevalecieran los mecanismos periféricos citados en los apartados 1,2 y 3 y aumentados cuando la patogenia del hiperglucocorticismo fuera central. Según Jeffcoate (8) los cuadros de Pseudo-Cushing alcohólico estarían condicionados más frecuentemente por estímulo hipotálamo-hipofisario y mostrarían, por tanto, cifras de ACTH elevadas. Con todo, no serían infrecuentes las hipercortisolemias con valores disminuidos de ACTH cono han descrito Chalmers, Freinkel, Merry, Wöber y Wright (1014) entre otros.

Por todo lo referido anteriormente nos propusimos determinar el valor que podrían tener estas determinaciones como marcadores de alcoholismo en pacientes alcohólicos ingresados en centros hospitalarios con clínica instaurada y en plena fase de abuso alcohólico o tras cortos períodos de abstinencia, que no sobrepasaban en sus límites máximos una semana.

\section{MATERIAL Y MÉTODOS}

Se estudiaron 24 pacientes alcohólicos ingresados con un cuadro de delirio agudo o con patologías no relacionadas directamente con el alcohol. El $62 \%$ de ellos habían bebido hasta el mismo día del ingreso y el resto habían dejado de beber durante un período inferior a una semana antes de su recepción en el hospital.

Las edades de los pacientes oscilaban entre 36 y 71 años. Edad media 49,6.

Junto a la determinación de cortisolemia a.m. y p.m. y de ACTH a.m. se valorarón además los siguientes parámetros relacionados con el alcohol:

- Serie roja con sus indices de Wintrobe.

-Número de plaquetas $/ \mathrm{mm}^{3}$.

- Trigliceridemia.

- Colesterolemia total y colesterolemia HDL.

-Niveles de actividad sérica de transaminasas glutámicooxal-acética y glutámico-purúvica.

- Valores en el suero de fosfatasa alcalina.

- Actividad sérica de la gamma-glutamil-transpeptidasa.

Aunque se refiere últimamente la utilidad como parámetro de etilismo de la determinación de CDT (transferrina deficiente en carbohidratos) (15), se ha renunciado a este método por no ser más útil que los usados por nosotros $(16,17)$ o incluso, según otros autores (18), por ser menos eficiente que los referidos.

\section{RESULTADOS}

El consumo medio de alcohol de nuestros pacientes era de 178 gramos/día en los periodos de tiempo más inmediatos a su ingreso.

En relación con los datos analíticos de alcoholismo se demostró:

1. El $82 \%$ de los pacientes ofrecía índices de Wintrobe elevados.

2. El $56 \%$ de la muestra mostraba cifras de plaquetas inferiores a $150.000 / \mathrm{mm}^{3}$ con valores mínimos de 62.000 y máximos de 463.000 (media de 190.696 y desv típ de 109.725).

3. Sólo en un $12,4 \%$ de los enfermos se demostró una trigliceridemia superior a $150 \mathrm{mg} \%$ para unos valores medios de 112,7 y una desv típ de 38,7.

4. La colesterolemia total estaba por debajo de $200 \mathrm{mg} \%$ en el $66,6 \%$, entre 200 y $250 \mathrm{mg} \%$ en el $14,3 \%$ y por encima de $250 \mathrm{mg} \%$ en el $19,1 \%$ de los casos para unos valores medios de $196 \mathrm{mg} \%$ y una desv típ de 86.66.

5. El colesterol-HDL se mueve en límites normales (entre 35 y $65 \mathrm{mg} \%$ ) en un $47 \%$, está descendido en otro $47 \%$ y alto sólo en el 6\% de la muestra con cifras medias de 38,6 y desv típ de 16,5 .

6. El $60,8 \%$ de los pacientes revelaban valores altos de TGO y/o de TGP en relación con una hepatopatía alcohólica concomitante.

7. Para valores normales de fosfatasa alcalina oscilantes entre 98 y $280 \mathrm{U} / \mathrm{l}$, solamente el $21,7 \%$ de nuestros pacientes la presentaban elevada (media de 210,95 y des típ de 108,12).

8. Solamente el $17,3 \%$ de los enfermos alcohólicos ofrecía cifras normales de gamma-glutamil-transpeptidasa (11-50 U/l) y el resto $(82,7 \%)$ valores claramente elevados (media de 222.21 y desv. típ. de 248,92).

9. La cifra media de ACTH a.m. fue de $41,2 \mathrm{pg} / \mathrm{ml}$ para valores normales de 10-80 pg/ml. Sólo en una ocasión se observó una ACTH alta $(146 \mathrm{pg} / \mathrm{ml})$, pero acompañada de cortisolemias a.m. y p.m. absolutamente normales.

10. El valor medio de la cortisolemia a.m. fue de 22 $\mathrm{mcg} / \mathrm{ml}$ (rango normal: $5-25 \mathrm{mcg} / \mathrm{ml}$ ) y el de cortisolemia p.m. de $12,3 \mathrm{mcg} / \mathrm{ml}$ (rango normal: $5-15 \mathrm{mcg} / \mathrm{ml}$ ). Un caso ofrecía una elevación mínima simultánea de 33 y $17 \mathrm{mcg} / \mathrm{ml}$ de cortisolemia a.m. y p.m. respectivamente con valores de ACTH normales.

\section{DISCUSIÓN}

Ninguno de nuestros pacientes alcohólicos mostraba rasgos clínicos de síndrome pseudocushingoide y los valores medios de cortisolemia y de ACTH en sangre resultaron normales. Sólo un enfermo presentó tasas elevadas de ACTH con cortisolemias a.m. y p.m. normales y otro ofreció un mínimo ascenso de las cortisolemias a.m. y p.m. con ACTH normal. Las explicaciones posibles a estas observaciones serían:

- Los niveles de ingestión de alcohol no eran lo suficientemente altos como para inducir una hiperfunción directa de las suprarrenales o a través del eje hipófiso-suprarrenal. 
Dado el alto consumo de alcohol de nuestros enfermos (178 $\mathrm{g}$ /día), no parece útil el considerar el que la ingestión diaria fuera aún superior.

b) El síndrome pseudocoshingoide de estos pacientes alcohólicos podría ser subclínico y mostrarse solamente en forma de unos niveles de cortisolemia elevados, que se normalizarían tras la abstención alcohólica, como ya refirieron en 1976 Smals y cols. (3), Frajria y Angeli (5) si bien estos autores afirman que el período de normalización es de unas pocas semanas sin concretar más con respecto al tiempo.

Según nuestras observaciones para los consumos de alcohol de los pacientes, que eran, como se ha detallado, francamente elevados, cabe afirmar que el síndrome de Pseudo-Cushing es excepcional. Jeffcoate (8) ya había insistido en que el Pseudo-Cusing alcoholico no era frecuente y en que había que distinguirlo del aspecto cushingoide de muchos alcohólicos, en los que síntomas como hipertensión, miopatía, inyección conjuntival, osteoporosis, piel fina y depresión estaban determinados por la acción periférica del alcohol sin real alteración funcional suprarrenal, ya que estos enfermos mostraban una eliminación diaria de cortisol libre urinario normal. Incluso los casos en los que la cortisolemia libre estaba elevada, esto era atribuible a la disminución de la síntesis de albúmina y de la CBG (globulina transportadora de cortisol), que normal- mente vehicula el $95 \%$ del cortisol sanguíneo, con lo que aumentaría el cortisol libre circulante sin haber realmente una hiperfunción cortical. Este fenotipo cushingoide es descrito también por Huerta y cols. (19) sin hiperfunción real del eje hipófiso-suprarrenal y explicado por la acción directa del etanol sobre los receptores periféricos.

Los enfermos estudiados por nosotros tampoco mostraron signos sugestivos de fenotipo cushingoide, que según Huerta y colaboradores, cuando se instauran, empiezan a remitir después de dos semanas de abstinencia alcohólica, período superior al que presentaban nuestros pacientes.

Concluimos, pues, que ni el síndrome ni el fenotipo cushingoides son observaciones frecuentes en los alcohólicos ni las determinaciones de ACTH y de cortisolemia a.m. y p.m. tienen utilidad alguna como marcadores de alcohol tras cortos períodos de abstinencia y con mucha más razón cuantos estos son largos.

Del resto de los parámetros destacan la gamma-glutamiltranspeptidasa con aumento en el $82,7 \%$ de las muestras y los índices de Wintrobe elevados $(82 \%)$. La plaquetopenia fue observada en el $56 \%$ de los enfermos. La colesterolemia total, el HDL-colesterol, la trigliceridemia y la actividad de fosfatasa alcalina en el suero se revelan como marcadores poco útiles en el alcoholismo.

\section{Bibliografía}

1. Kissin B, Schenker V, Schenker AC. The acute effect of ethanol ingestion on plasma and urinary 19 hidroxycorticoids in alcoholic subjects. Am J Med Sci 1960; 239: 690-704.

2. Kalant H, Hawkins RD,Czaja C. Effect of acute alcohol intoxication on steroid output of rat adrenals in vitro. Am J Physiol 1963; 204: 849-855.

3. Smals AG, Kloppenborg PW, Njo KT, et al. Alcohol induced cushingoid syndrome. Br Med J 1976: 1298

4. Rees LH, Besser GM, Jeffcoate WJ, et al. Alcohol-induced pseudo-Cushing syndrome. Lancet 1977: 726-8.

5. Frajria R, Angeli A. Alcohol induced Pseudo-Cushing syndrome. Lancet 1977: 1050-1.

6. Cubelos Pascual MI. Efectos del alcohol sobre las glándulas suprarenales de la rata.Estudio micrográfico.Tesis Doctoral.Universidad de Oviedo, 1994.

7. Coob CF, Van Thiel DH, Gavaler JS, et al. Effects of ethanol and acetaldehyde on the rat adrenal. Metabolism 1981; 30: 537-43.

8. Jeffcoate W. Alcohol induced Pseudo-Cushing syndrome. Lancet 1993; 341: 676-7.

9. Kapcala LP. Alcohol-induced Pseudo-Cushing's syndrome mimicking Cushing's disease in a patient with a adrenal mass. Am J Med 1987; 82: 849-56.

10. Chalmers RJ, Bennie EH, Johnson RH, et al. The growth hormone response to insulin induced hypoglycemia in alcoholics. Psychological Med 1978; 7: 607-11.

11. Freinkel N, Singer DL, Arky RA, et al. Alcohol hypoglycemia.Carbohydrate metabolism of patients with clinical alcohol hypoglycemia and the experimental reproduction of the syndrome with pure ethanol. $\mathrm{J}$ Clin Invest 1963; 42: 1112-33.

12. Merry J, Marks V. The effect of alcohol,barbiture and diazepam on hypothalamic-pituitary -adrenal function in chronic alcoholics. Lancet 1972: 990-2.

13. Woeber KA, Arky R. Hypoglycemia as the result of isolated corticothrophin deficiency. Br Med J 1965: 857-8.

14. Wright JW. Pituitary function in chronic alcoholism, M. Sc. Thesis.University of Surrey.

15. Fletscher LM. Alcohol and iron :one glass of red or more? J Gastroenterol Hepatol 1996; 11: 1039-41.

16. Rubio C, Gil V, Aparicio JM, et al. Eficacia diagnóstica de los marcadores biológicos de consumo de alcohol para la detección de abuso alcohólico. An Med Interna (Madrid) 1996; 13: 274-8.

17. Sharpe PC, McBride R, Archbold GP. Biochemical markers of alcohol abuse. QJM 1996; 89: 137-144.

18. Santo Domingo J, Rubio G, Marín JJ, et al. Transferrina pobre en hidratos de carbono y otros marcadores de consumo de alcohol en un hospital general. Rev Clin Esp 1997; 197: 627-30.

19. Mendoza H, Gorriz B, Lozano A, et al. Pseudocushing inducido por el alcohol v.s. fenotipo cushingoide. An Med Interna (Madrid) 1998; 15: 172-3. 